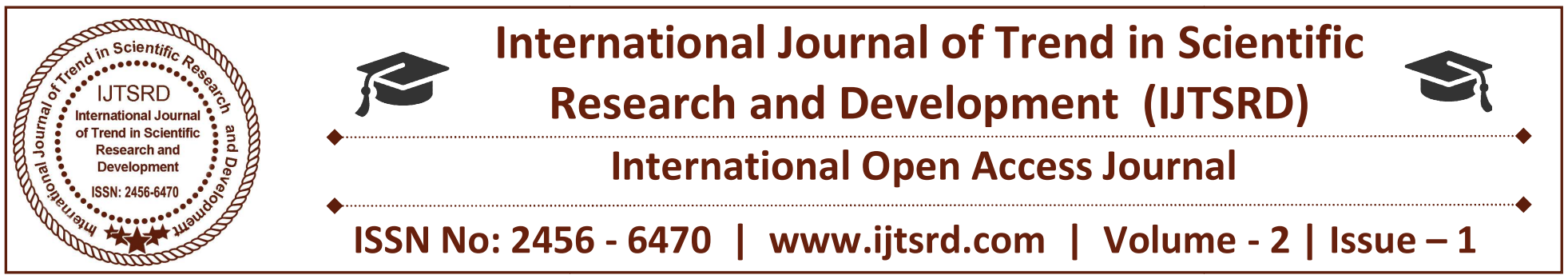

\title{
Soft Computing Healthcare Intelligence through Multisensory Activity Recognition
}

\author{
S. M. Sivakama Sundari \\ M.Tech, 2nd year Communication Systems, \\ Prist University, Chennai, Tamil Nadu
}

\author{
Dr. A. Rijuvana Begum \\ M.E, Ph. D, H.O.D of ECE Department, Prist \\ Univetsity, Chennai, Tamil Nadu
}

\begin{abstract}
Remote patient monitoring through telemedicine has become very usual nowadays. Sensor are attached to the patient's body and they monitor the vital signs during various activity, the data acquires from sensor is given as input to genetic algorithm based activity recognition system. If any deviations are found in vital signs during any activity, it is informed to the physician. Simulation was performed by taking 10 sample patients and some activities. Results prove the efficiency of the algorithm.
\end{abstract}

\section{INTRODUCTION:}

Telemedicine plays a very important role in patient management and have been effectively used for intra hospital transport of patients. Live monitoring of patients from both the hospitals creates new challenges. Similarly issues arise as how to process the data captured in real time. Sensors can overcome some of the challenges faced in telemedicine (Gaynor et al 2004). Advances in Micro Electro Mechanical Systems (MEMS) and Nano technology have enabled design of low powers sensor nodes capable of sensing different vital signs in our body. These nodes can communicate with each other to aggregate data and transmit vital parameters to a Base Station. The data collected in the base station can be used to monitor health in real time. The patient wearing sensors may be mobile leading to aggregation of data from different BS for processing. Processing real time data is compute intensive and telemedicine facilities may not have appropriate hardware to process the real time data effectively. Hence we propose a genetic algorithm based activity recognition which monitors the patient activity and send message to the doctor and aids in medication. Fusion weight prediction plays an important rule and GA has been used to find the fusion classifier.

\section{Sensors\& telemedicine:}

Sensor like gyro-scope [5], motion sensor [7], magnetometer [5], [6], accelerometer [2], [3], [4] microphone [4], [6], [7], barometer [8], light [6], [4] temperature [2], [6], RFID [9], etc. There are two types of activity recognition vision object sensor based activity recognition and on body sensor based activity recognition.

In the first method sensors are attached to objects that a person is using. Normally sensors were attached to furniture to infer activities [10]. RFIDs identify the patient's detailed activities such as put on lotion, taking photo, etc. [9]. This method uses the semantic relationships between patients and activities to classify activities. However, a large number of sensors are required which is infeasible and time consuming process, uncertainty of sensors such as false start and unable to detect. Object could also result in a poor recognition rate

The second method collects data e.g. movement from sensors attached on human body. Common sensors are accelerometer and inertia sensor. The sensors are attached to as chest [10], wrist [2], waist [3], etc. However, sensors are required to be worn at all-time which may interrupt or reduce mobility of a user or even obstruct daily activities routine.. It is important that the activity recognition model is highly accurate and also practical. Recent work [2], [4] showed an 
activity recognition model which is practical and highly accurate based on wrist-worn sensors. Accelerometer, temperature sensor and altimeter were used and 90\% accuracy was achieved.

In addition, heart rate monitor, barometer and light sensor are also used. Heart rate can be used to measure physical activities indirectly as heart rate is proportional to the intensity of movement and oxygen supplied to skeletal muscles [11]. A combination of acceleration and heart rate improve accuracy of estimation of energy expenditure by $1.4 \%$ [12]. However, the study concluded that the small improvement was not worth it as the user needs to wear the heart rate monitor at all times. Accelerometer and barometer (air pressure differential) were used to detect ambulatory movements considering vertical position shifts [8]. Combining barometer and accelerometer improved classification accuracy in child activities [3].

Temperature could be used to indicate changes in environment when performing certain activities e.g. washing dishes and brushing teeth may involve a use of water or when ironing, the temperature maybe higher than normal. Several works used the temperature sensor as part of their activity recognition systems [6], [7]. For example, the difference of temperature of 15 minutes was used to determine the use of a shower [7]. Work by [4], [6] used light sensors as part of their activity recognition systems. Gyroscope can be used to estimate the orientation and rotation of the movement. After gyroscope and magnetometer were added to the accelerometer, the accuracy was increased by $17 \%$ [5]. From literatures, it can be seen that combining these sensors could improve classification accuracy. However, these sensors have not yet been combined on the wrist location.

\section{Methodology:}

In real applications, we expect that the sensors will be embedded in a small wearable component and patient it is attached inpatient's wrist. Multiple sensors on the dominant wrist. Are sent wirelessly to the PC. The activity recognition model performs a classification and stores the results on PC. The predicted activity is encrypted and sent over the Internet to the patient's doctor and family member. The stakeholders PCs or mobiles should contain specialized models for further analysis e.g. to generate behaviour pattern for health professionals, to show a day activity report for family members, etc. The detected activity can also be used to provide services in homes. For example, if a raise in temperature is found, the doctor may advice medication. The various prediction algorithm used are

1) Multi-Layer Perceptron neural network (MLP) MLP uses the concept of connectionist where several input nodes are connected with associated weights to several outputs nodes. The network output can be calculated from the summation function oi $=\varphi(-i$ Wixi) where $\mathrm{Wi}$ is the weight used for adjusting input $x i$ and $\varphi$ is the activation function [13]. MLP learns the classification error through a back propagation algorithm and tries to find the weights to minimize

2) Radial Basis Function neural network (RBF) RBF [13] is a neural network which uses RBF as an activation function. For $\mathrm{N}$ hidden neurons, the activation function is $\mathrm{f}(\mathrm{x})=\mathrm{Ni}=1 \mathrm{Wi \phi}(\mathrm{x}-\mathrm{ci})$ where ci is the centre vector for neuron $i$ and $\phi$ is a kernel function.

\section{3) Support Vector Machine:}

Support Vector Machine (SVM) SVM [18] constructs decision boundaries by solving the optimization objective $\min \mathrm{W}, \mathrm{b}, \xi \quad 1 \quad 2 \mathrm{WTW}$ $+\mathrm{C} \longrightarrow \mathrm{m} \mathrm{i}=1 \quad \xi \mathrm{i}$ subject to yi(WTf(xi)+b) $\geq 1-\xi i$ and $\xi \mathrm{i} \geq 0$ The slack term $\xi \mathrm{i}$ is used to relax the constraints allowing misclassified examples. The associated cost parameter $\mathrm{C}$ is used for penalizing $\xi i \mathrm{f}()$ is a function which transforms the input xi into a higher dimensional space. This study used a RBF kernel function $f(x i)=\exp (-1(2 \sigma 2) x i-x j$ 2) where $\sigma$ is the width of the Gaussian kernel. For multiclass classification, we constructed $\mathrm{K}$ binary classifiers and applied one-VS-all classification.

Since each classification model may be superior to others, it is common to incorporate weights to the models to reflect this. Six weight functions are studied. Simple average (SA) gives the average weights to all classifiers. Variance-covariance (VACO) uses the mean square error to calculate the weights. In this study we modified the VACO equation to suit a classification problem by utilizing class probabilities. Discounted mean square forecast error (DMSFE) is the modified version of VACO where a parameter $\beta$ is used to discount weights of the instances. Unit weight gives all classifier weights 1 which means all classifiers are associated with weights. Weighted accuracy (WACC) uses weighted 
accuracy of each model as the weights. Note that all calculated weights must be summed to one

\section{Proposed Genetic Algorithm based algorithm}

In this study, we propose to use GA to find weights for classifiers. GA [19] has been commonly used to solve an optimization problem. The advantage of GA over other optimization techniques is that instead of starting at a single point to find the solution, a population of points is created. It mimics natural selection in which the population is modified over time. Individuals are randomly selected as parents to produce children of the next generation. 1) Fitness function: GA is used to find the weights that minimize the mean square of the combination error. The classification error is defined

$$
\text { error }_{i}=\left\{\begin{array}{l}
1 \\
0
\end{array}\right.
$$

If truei=prei

The fitness function is

$$
f f\left(w_{j}\right)=\frac{1}{2 m} \sum_{i=1}^{m} \operatorname{error}\left(\operatorname{tru}_{i}, f m_{i}(w\right.
$$

Contribution of a sensor in the network We use two techniques to investigate the importance of the sensor i.e. mutual information to measure the importance of the sensor to the classification and Clamping to measure the importance of the sensor within the model. 1) Mutual information (MI) [20] MI is based on information theory. It is used for defining the dependency between variables. Given two variables, $\mathrm{x}, \mathrm{y}$, the mutual information can be calculated as $\mathrm{I}(\mathrm{x} ; \mathrm{y})=\mathrm{p}(\mathrm{x}, \mathrm{y}) \log \mathrm{p}(\mathrm{x}, \mathrm{y}) \mathrm{p}(\mathrm{x}) \mathrm{p}(\mathrm{y}) \mathrm{dxdy}$. 2) Clamping [16] MLP is constructed using several sensors based on the feature selection process. Features of each sensor are substituted using their mean values. If the sensor is important in the network, removing it would result in lower network performance. Assuming all features within a sensor give equal significance, the contribution of a particular sensor is $\operatorname{con}(\mathrm{S})=1-$ $\mathrm{g}\left(\mathrm{F} \mid \mathrm{S}={ }^{-} \mathrm{S}\right) \mathrm{g}(\mathrm{F})$ where $\mathrm{F}$ is a set of features, $\mathrm{S}$ is the set of features of a particular sensor, $g\left(F \mid S={ }^{-} \mathrm{S}\right)$ is the performance of the network when the values of $S$ are substituted by their mean values, and $g(F)$ is the generalized performance. The schematic diagram KS given below in figure 1

The average classification results using different classifier fusion methods and fusion weight

\begin{tabular}{|l|l|l|}
\hline & Accuracy in \% & improvement \\
\hline Best individual & 96 & \\
\hline Majority vote & 97.15 & .13 \\
\hline product & 97.24 & .11 \\
\hline sum & 97.31 & .12 \\
\hline min & 97.12 & .9 \\
\hline max & 97.16 & .17 \\
\hline ranking & 96.86 & .89 \\
\hline
\end{tabular}

SA $97.2078 \pm 0.53910 .241713 .45030 .000086 .5497$

VACO 97.2141 \pm 0.57230 .24796 .14043 .508890 .3509$

DMSFE-0.80 96.9824 \pm 0.64280 .016228 .36260 .584871 .0526$

DMSFE-0.85 $97.0493 \pm 0.61150 .083126 .02340 .584873 .3918$

DMSFE-0.90 97.1451 \pm 0.57800 .178916 .37430 .584883 .0409$

DMSFE-0.95 97.2298 \pm 0.56100 .263612 .57310 .292487 .1345$ 
No weight $97.1974 \pm 0.54280 .231214 .03510 .000085 .9649$ Weighted accuracy $97.2924 \pm 0.4870$

0.32627 .01750 .000092 .9825 GA-function $97.1178 \pm 0.33460 .15162 .924012 .573184 .5029$ GAlinear

\begin{tabular}{|l|l|l|}
\hline & Accuracy in \% & improvement \\
\hline SA & 96 & .9 \\
\hline VACO & 97.15 & .79 \\
\hline DMSFE-0.80 & 97.24 & .89 \\
\hline DMSFE-0.85 & 97.31 & .87 \\
\hline DMSFE-0.90 & 97.12 & .834 \\
\hline DMSFE-0.95 & 97.16 & .921 \\
\hline No weight & 96.86 & .13 \\
\hline GA-function & 94.65 & .11 \\
\hline GA-function & 96.38 & .12 \\
\hline
\end{tabular}

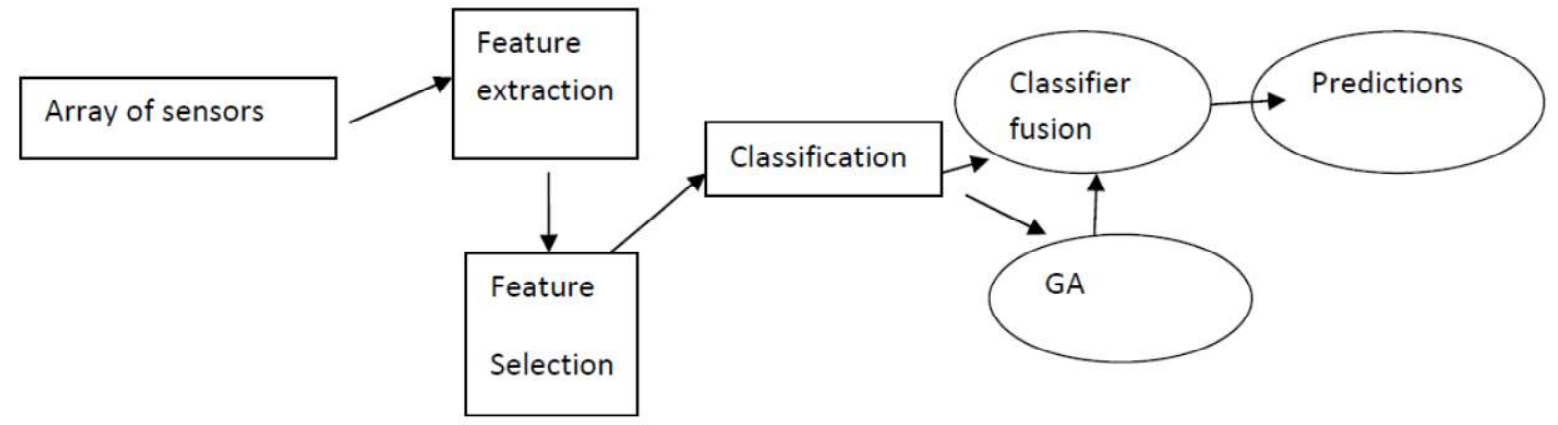

FIGURE 1

\section{RESULTS AND DISCUSSION:}

The participants were first interviewed on their gender, age, and health issue to evaluate their suitability for participation. 12 participants were recruited for the study including two males and ten females aged $72.55 \pm 4.321$ years. During the simulation period, patients were instructed to perform activities including brushing teeth, exercising, feeding, ironing, reading, scrubbing, sleeping, using stairs, sweeping, walking, washing dishes, watching TV and wiping. The participants were asked to perform each activity for 10 minutes. They were allowed to perform the activities in any order and could take breaks during

A total of fifty hours of activity of patients data were collected. All missing data were removed. Also, to keep the balance between classes, sweeping floor activity data were removed as after removing missing data it only constitutes to $5 \%$ of the dataset. The data was pre-processing using WMA where w1 was set to 0.3 and w2 was set to 0.7 in the experiment. The data were segmented at 3.5 seconds with $40 \%$ overlapping. The data set contains 5000 samples. The features are calculated as mentioned in Section II-D. NaN and constant valued features were removed. To reduce the feature space, we examined the MI of each feature. Using a cut-off point at 5\% of MI, the number of features is reduced from 150 to 90 . All experiments in this study used 10-fold cross validation where 7 folds were used for training, 1 for validation and 2 for testing. The data were randomly selected with equal class distributions. All experiments were executed 50 runs. 


\section{Importance of sensors and suggested features:}

The importance of sensors and features were analyzed using MI. The result shows that accelerometer was the most important sensor. $34 \%$ of accelerometer features contained over 3rd quartile of MI about the classes. Altimeter and temperature sensors were the least important sensors. Gyroscope, barometer and light were also among the most important sensors containing useful information in classifying 17activities. Accelerometer and gyroscopes produce the top ten MI (See Table III). MI of some of the features calculated from these sensors was in the 3rd quartile or higher. Also, it can be seen in Table IV that the time domain features provide more useful information than frequency domain features. Maximum, RMS, mean, median, STD, mode, minimum, intensity were the most important features, respectively. The feature selection was performed using FC. The truncation at 24 features was selected as the accuracy started to remain constant. Features from accelerometer, altimeter, and heart rate monitor light and barometer were selected. Also, 16 features were used to conform to previous study. Next, the contributions of sensors in our model (with 24 features) were investigated. The result shows that accelerometer was the most important sensor in the model. This is followed by altimeter, heart rate monitor (HR), barometer, gyroscope, and light respectively. The top three features with the highest importance in the model were mean acceleration on $\mathrm{Z}$-axis, maximum barometer pressure, and minimum altitude, respectively.

B. Classifier fusion Classification was performed using 3 algorithms with 18 and 26 features. In total, 6 classification models were produced which gave mean accuracy between $90.45 \%$ and $94.14 \%$ with STD between 0.3088 and 0.4186 . As expected, SVM performance was superior to other algorithms. However, according to precision and recall of each classifier, some classifiers were better than SVM in some of the activities. Next, classifier fusion was performed. Data from training and validation set were used to determine the weight for SA, VACO, DMSFE and WACC techniques, whereas in GAFW, the training set was used in the fitness function and the validation set was used to select the weight. There are 57 possible combinations which were generated from the 6 classifiers. The results of the classifier fusion on the test data are presented in Table I and Table VI. The classifiers fusion result is compared with the best individual classifier (BI) within the fusion group. The improvement column shows the percentage of mean difference between classifier fusion and BI. It can be seen that classifier fusion which utilized posterior probability achieved better results comparing to fusing the class output directly. Among seven classifier fusion methods, sum was the best fusion technique. It improved classification accuracy by $0.3435 \%$ on average comparing to using only the best individual classifier. $95.79 \%$ of all possible combinations using the sum method achieved equal or higher accuracy than using the best classifier

The average classification results using different classifier fusion methods and fusion weight

\begin{tabular}{|l|l|l|}
\hline & $\begin{array}{l}\text { Accuracy in } \\
\%\end{array}$ & Improvement \\
\hline Best individual & 96 & .13 \\
\hline Majority vote & 97.15 & .11 \\
\hline Product & 97.24 & .12 \\
\hline Sum & 97.31 & .9 \\
\hline Min & 97.12 & .17 \\
\hline Max & 97.16 & .89 \\
\hline Ranking & 96.86 & using \\
\hline
\end{tabular}

The average classification results using different fusion weight functions

\begin{tabular}{|l|l|l|}
\hline & $\begin{array}{l}\text { Accuracy in } \\
\%\end{array}$ & .9 \\
\hline SA & 96 & .79 \\
\hline VACO & 97.15 & .89 \\
\hline DMSFE-0.85 & 97.24 & .87 \\
\hline DMSFE-0.90 & 97.12 & .834 \\
\hline DMSFE-0.95 & 97.16 & .921 \\
\hline No weight & 96.86 & .13 \\
\hline GA-function & 94.65 & .11 \\
\hline GA-function & 96.38 & .12 \\
\hline
\end{tabular}


The result of the study indicates that accelerometer is the most important sensor for activity recognition. This confirms that accelerometer has ability of measuring human activity quantitatively, fast reaction to changes in movement and reflects type of activity well [6]. We also find that the new sensors introduced including gyroscope, barometer and light contain useful information about human activities. Similar to accelerometer, gyroscopes can reflect changes in activity well. We also observe that data obtained from gyroscope are similar to those from accelerometer. Barometer and light can be used to differentiate activities such as using stairs and sleeping. Interestingly, although gyroscope, barometer and light are shown to be very important sensors on their own, this is not the case when they are combined together. In our model of 24 feature selected using FC. Also, its contribution to the network is not as high as other sensors. This may be explained that although gyroscope is a good sensor on its own, when it is used with accelerometer, many of its features become redundant. The result also indicates that heart rate has significant contribution to the model. Using heart rate in the model increases the accuracy by $1.74 \%$. The statistical tests showed that the improvement is significant $(\mathrm{p}<0.05)$. This may be due to the fact that majority of activities studied in [12] are exercise related activities e.g. cycling, running, rowing, etc.

Although, heart rate help improve exercise activities, due to the similarity in these activities and large number of classes, the overall improvement is not as high as they expected. On the other hand, our study contains activities which are rather different e.g. walking, sleeping, exercise, large difference in heart rate between these activities are expected and thus resulting in heart rate having a significant impact in our model. Several classifier fusion and fusion weight techniques were investigated. The results show sum is the most effective fusion method and when used with SA, WACC, or GA, improvement on all combinations can be achieved. As sum technique uses the average probability, the result is not heavily affected when some classifiers are over confident. On the contrary, min method selects the class that has the minimum objection by all classifiers. As min is sensitive toward objection, it is affected when some inaccurate classifiers always produce low probability. Similarly for max technique, if the system contains bad classifiers that produce high probability, the system accuracy is affected. The results show ranking is the worst fusion method. Although ranking reduces the bias caused by some classifiers being over confident, converting probabilities into rank also loses some information. Thus, fusing classifiers could produce conflict or wrong prediction if there are many inaccurate classifiers in the group.

Product technique shows the best result when unit weight is used The results of the study also showed that using GA to find the fusion weight uses a much higher computational cost than other functions especially when trying to optimize min and max function. Therefore, the proposed GAFW should be appropriate in the activity recognition model that will be developed offline. For other system that needs to update the fusion weights in real time, other functions such as VACO and WACC should be used. For the classifier combination function, the computational cost is very low and can be apply in both online and offline applications.

\section{CONCLUSION:}

The proposed GA can be applied with any systems aim to combine multiple classifiers. This study has demonstrated that $98 \%$ of classifier fusion using GA achieves higher accuracy than using only the best classifier. While other fusion weight techniques cannot guarantee accuracy improvement, we show that GA is a more suitable method for determining fusion weight for activity recognition using sensors regardless which fusion techniques

\section{REFERENCES}

1) Gaynor, M.; Moulton, S.L.; Welsh, M.; LaCombe, E.; Rowan, A.; Wynne, J.; Integrating wireless sensor networks with the grid Internet Computing, IEEE Issue Date: July-Aug. 2004 Volume: 8 Issue:4 On page(s): 32 - 39

2) S. Chernbumroong, S. Cang, A. Atkins, and H. $\mathrm{Yu}$, "Elderly activities recognition and classification for applications in assisted living," ExpertSystems with Applications, vol. 40, no. 5, pp. $1662-1674,2013$.

3) Y. Nam and J. Park, "Child activity recognition based on cooperative fusion model of a triaxial accelerometer and a barometric pressure sensor," Biomedical and Health Informatics, IEEE Journal of, vol. 17, no. 2, pp. 420-426, 2013.

4) U. Maurer, A. Rowe, A. Smailagic, and D. Siewiorek, "Location and activity recognition using ewatch: A wearable sensor platform," 
AmbientIntelligence in Everyday Life, pp. 86-102, 2006.

5) H. Gjoreski and M. Gams, "Activity/posture recognition using wearable sensors placed on different body locations," Proceeding of signal andimage processing and applications, 2011.

6) J. Parkka, M. Ermes, P. Korpipaa, J. Mantyjarvi, J. Peltola, and I. Korho-nen, "Activity classification using realistic data from wearable sensors," Information Technology in Biomedicine, IEEE Transactions on, vol. 10,no. 1, pp. 119-128, 2006.

7) A. Fleury, M. Vacher, and N. Noury, "Svm-based multimodal classi-fication of activities of daily living in health smart homes: Sensors, algorithms, and first experimental results," Information Technology inBiomedicine, IEEE Transactions on, vol. 14, no. 2, pp. 274-283, 2010.

8) Y. Ohtaki, H. Inooka, K. Sagawa, A. Suzuki, Z. Xiumin, M. Okutsu, and R. Nagatomi, "Recognition of daily ambulatory movements utilizing accelerometer and barometer," Power, vol. 100, p. 102, 2004.

9) Y.-J. Hong, I.-J. Kim, S. C. Ahn, and H.-G. Kim, "Mobile health monitoring system based on activity recognition using accelerometer," Simulation Modelling Practice and Theory, vol. 18, no. 4, pp. $446-455,2010$.

10) P. Barsocchi, "Position recognition to support bedsores prevention," Biomedical and Health Informatics, IEEE Journal of, vol. 17, no. 1, 5359, 2013.

11) J. Booyens and G. Hervey, "The pulse rate as a means of measuring metabolic rate in man," Canadian Journal of Biochemistry and Physiology, vol. 38, no. 11, pp. 1301-1309, 1960.

12) E. Munguia Tapia, "Using machine learning for real-time activity recognition and estimation of energy expenditure," Ph.D. dissertation, Massachusetts Institute of Technology, 2008.

13)C. M. Bishop, Neural Networks for Pattern Recognition. New York, NY, USA: Oxford University Press, Inc., 1995. 